\title{
The Role of Computed Tomography Coronary Angiography in the Management of Coronary Anomalies
}

\author{
Silvia Tresoldi, M.D.,* Miriam Mezzanzanica, M.D., $\dagger$ Alessandro Campari, M.D., $\dagger$ \\ Diego Salerno Uriarte, M.D., $\ddagger$ and Gianpaolo Cornalba, M.D. $\S$ \\ * Servizio di Radiologia Diagnostica ed Interventistica, Azienda Ospedaliera San Paolo, \\ Milan, Italy; †Scuola di Specializzazione in Radiodiagnostica, Università degli Studi di Milano, \\ Milan, Italy; $\ddagger$ Reparto di Cardiologia, Azienda Ospedaliera San Paolo, Milan, Italy; and \\ $\S$ Dipartimento di Scienze della Salute, Università degli Studi di Milano, c/o Azienda \\ Ospedaliera San Paolo, Milan, Italy
}

\begin{abstract}
АвSTRACt Congenital abnormalities of coronary arteries are an uncommon cause of sudden cardiac death and are difficult to detect at coronary angiography. We describe two patients with acute coronary syndrome and non-occlusive coronary artery disease in which a 64-multidetector computed tomography (MDCT) coronary angiography showed the presence of a malignant coronary anomaly. Sixty-four-MDCT with the possibility of 3D reconstructions allows for easier diagnosis of coronary anomalies and provides essential details necessary for operative intervention. doi: 10.1111/jocs.12040 (J Card Surg 2013;28:33-36)
\end{abstract}

Congenital abnormalities of coronary arteries may be found incidentally in $0.3 \%$ to $1 \%$ of healthy individuals. ${ }^{1}$ They are an uncommon but important cause of chest pain, myocardial ischemia, and syncope and, above all, they carry a high risk for sudden cardiac death (up to $30 \%$ of patients), particularly before 35 years of age. ${ }^{2}$ The detection and characterization of these anomalies is crucial for the management of these patients. We describe two cases of patients with angina but nonsignificant coronary artery disease at angiography in which the coronary anomaly was diagnosed using a 64MDCT (Light Speed VCT, General Electrics, Milwaukee, WI). The usefulness of this non-invasive diagnostic tool in the diagnosis of the coronary anomalies is the subject of this clinical review.

\section{PATIENT 1}

A 66-year-old female with hypertension presented with sudden chest pain. The electrocardiogram (ECG) showed sinus rhythm with ST elevation in V2 and V3 leads. Blood tests revealed a slight increase of

Disclosures: none.

Address for correspondence: Dr. Silvia Tresoldi, M.D., Servizio di Radiologia Diagnostica ed Interventistica, Azienda Ospedaliera San Paolo, Via A. di Rudinì 8, 20142 Milan, Italy. Fax: 3902 50323393; e-mail: silvia.3soldi@gmail.com
T-troponine $(0.06 \mathrm{ng} / \mathrm{mL}$-normal values 0.01 to $0.03 \mathrm{ng} / \mathrm{mL})$. The patient underwent conventional coronary angiography (CCA) which revealed a myocardial bridge of the anterior descending artery and an anomalous origin of the right coronary artery (RCA), with no significant disease of the other epicardial vessels (Fig. 1a). Computed tomography coronary angiography (CTCA) confirmed the absence of significant atherosclerosis and the presence of a short intramyocardial path of the anterior descending coronary artery (Fig. 1C) and, in addition, revealed the aberrant origin of the RCA from the opposite coronary sinus (Fig. 1b). The patient, though informed about potential risks associated with this anomaly and available therapeutic options, refused any surgical intervention and agreed to long-term $\beta$-blocker medical therapy with a strict follow-up. She has remained asymptomatic at one-year follow-up.

\section{PATIENT 2}

A 49-year-old male presented with chest pain. ECG showed sinus rhythm with ST elevation in all the electrocardiographic leads; the echocardiogram was normal; blood T-troponine values were $0.05 \mathrm{ng} / \mathrm{mL}$. The patient underwent coronary angiography that showed non-occlusive coronary artery disease and revealed an aberrant origin of the dominant RCA from the left 


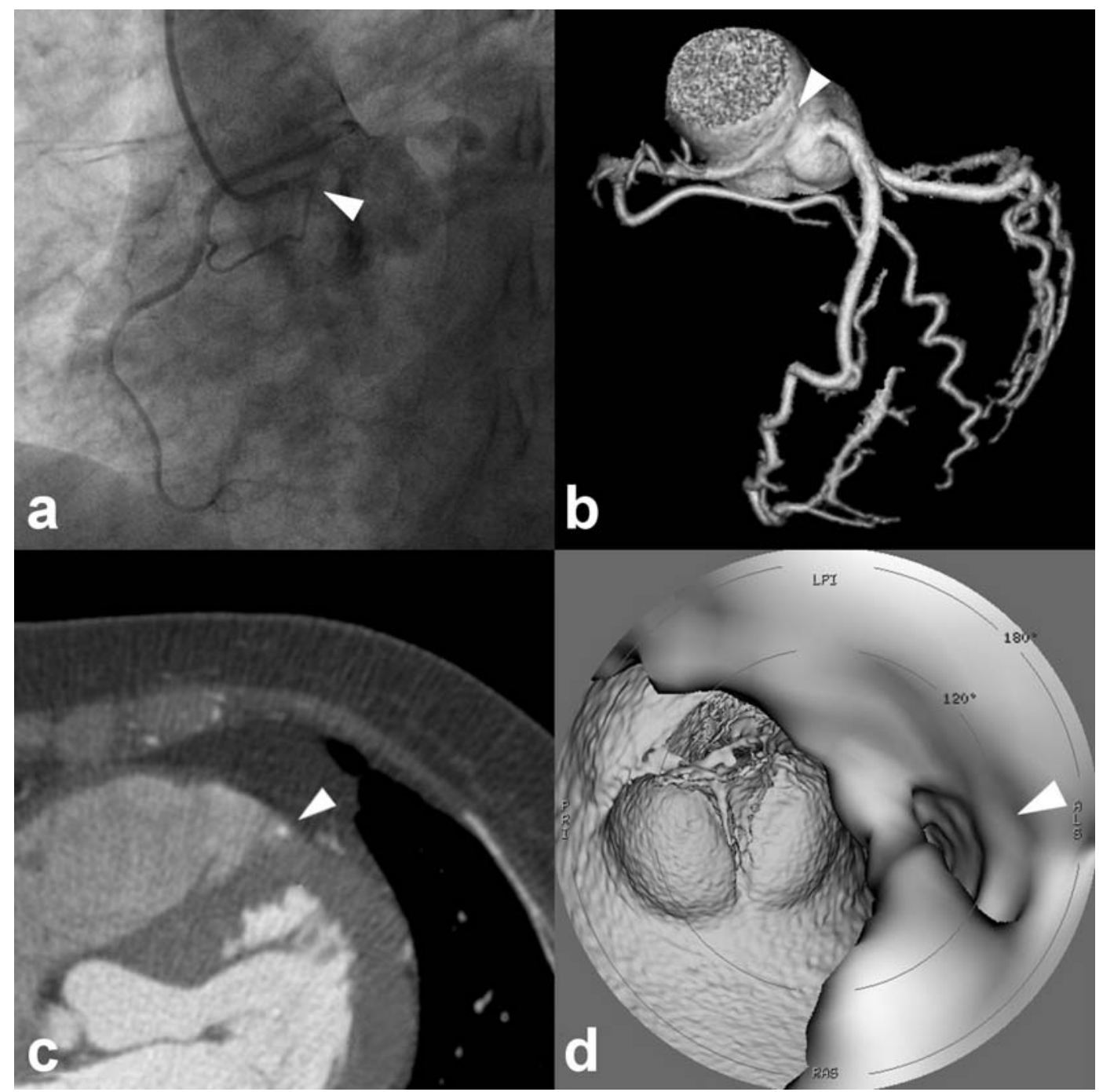

Figure 1. Sixty-six-year-old female patient with suspected acute coronary syndrome. Coronary angiography LAO image shows absence of significant stenosis of the right coronary artery (RCA). It is possible to suspect the anomalous RCA origin just above the left coronary artery (LCA) ostium (arrowhead in a). Computed tomography coronary angiography (CTCA) volume rendering image confirms the anomalous origin of the RCA, dominant, from the left coronary sinus (arrowhead in b) and the particularly tortuous path of LCA and its branches, as often occurs in hypertensive patients. CTCA maximum intensity projection (MIP) axial image depicts the intra-myocardial path of the anterior descending coronary artery (arrowhead in C). In CTCA 3D intraluminal view the ostium of the anomalous RCA is in the foreground and its oval shape is well depictable (white arrowhead in d); the aortic valve is visible in the background.

coronary sinus (Fig. 2a). CTCA showed the origin of the RCA from the left Valsalva sinus (Fig. 2b). This patient underwent surgery (venous graft between the aorta and the RCA) with complete remission of symptoms at six months follow-up. The patient was lost at one year follow-up because he moved to another country.

\section{DISCUSSION}

Congenital coronary artery abnormalities are an uncommon but important cause of sudden cardiac death. They are the most common cause of death during physical exercise in young adults and many patients have few symptoms before the onset of sudden cardiac death. ${ }^{3}$

Classification criteria for coronary anomalies are their origin, course, and termination. ${ }^{1}$ An anomalous coro- nary artery can rise from the opposite coronary sinus, from the noncoronary sinus or from the pulmonary artery and can take four different courses depending on the relationship of the vessel to the aorta and the pulmonary trunk: inter-arterial, retro-aortic, pre-pulmonic, and septal. In the inter-arterial course the proximal portion of the anomalous coronary artery goes between the aortic root and the pulmonary trunk, making it more prone to spasm. This anomaly carries a high risk for sudden death while the other courses seem to be benign. The variant seen in our two patients, with the RCA arising from the left Valsalva sinus and taking an inter-arterial pathway, is the most frequent and is associated with sudden cardiac death in up of $30 \%$ of patients. ${ }^{1}$ Increased risk of sudden cardiac death in patients with a RCA arising from the left coronary sinus has also been associated with an acute take-off angle of 


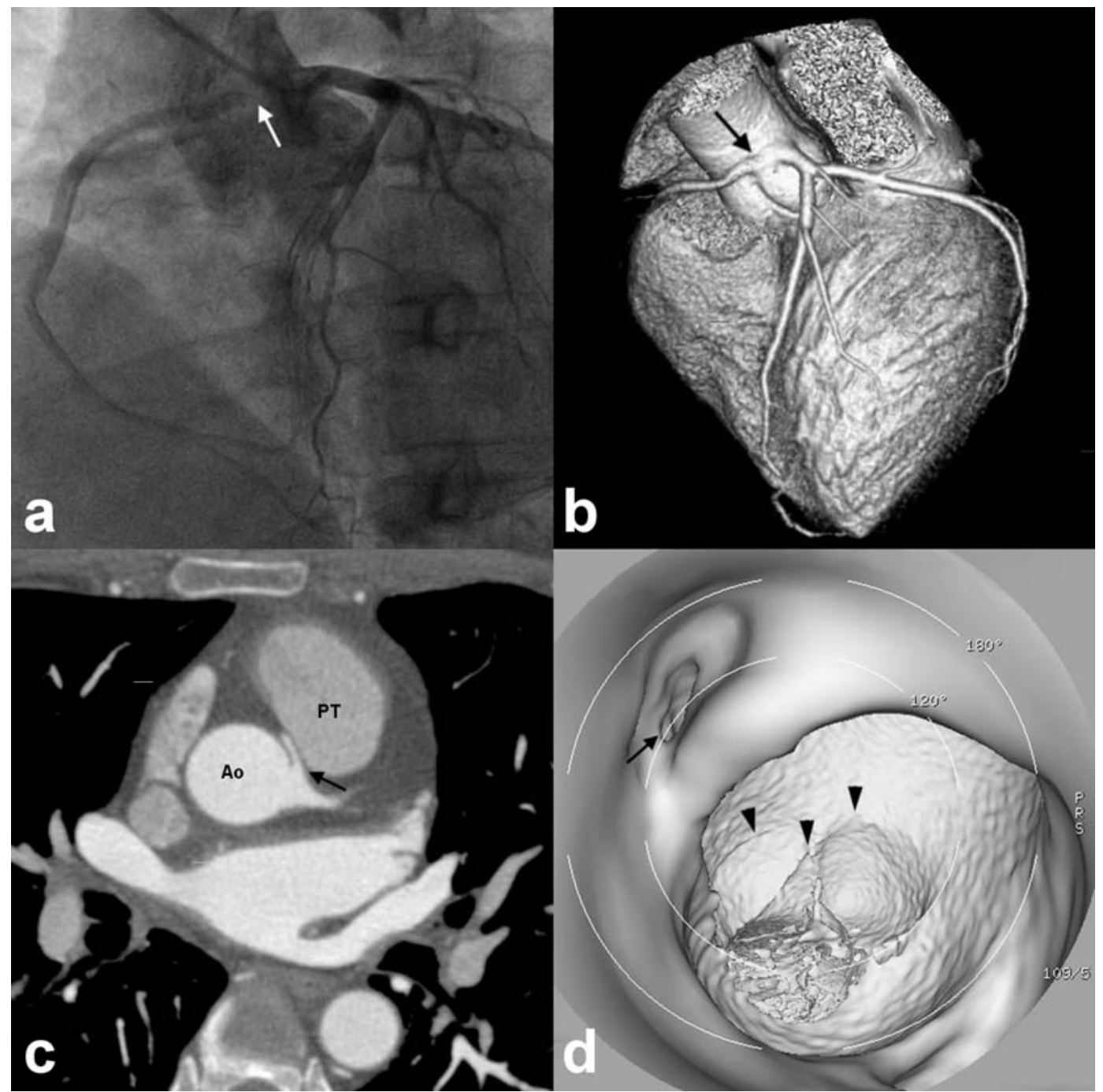

Figure 2. Forty-nine-year-old male patient with suspected acute coronary syndrome. Coronary angiography image shows the aberrant origin (arrow in a) of the right coronary artery (RCA), dominant, from the left coronary sinus and the absence of occlusive coronary artery disease. Computed tomography coronary angiography (CTCA) volume rendering image at the same view of the conventional angiography confirms the absence of significant stenosis in both coronary arteries and better clarifies the anomalous origin of the RCA from the left coronary sinus (arrow in b). CTCA maximum intensity projection (MIP) axial image shows the narrowed proximal tract of the anomalous RCA (arrow in c): the acute angle at the juncture between the vessel and the aortic wall and its malignant inter-arterial course are well-represented. CTCA 3D intraluminal view from the surgeon's perspective allows to evaluate the relationship between the aortic valve leaflets (arrowheads in d) and the oval shaped RCA ostium (arrow in d). Ao, aortic root; PT, pulmonary trunk.

the anomalous coronary artery from the aorta and with a slit-like coronary orifice that narrows when dilation of the aorta occurs during exercise, limiting blood flow. It is reported that CCA allows the correct identification of this kind of abnormality in only $53 \%$ of cases. ${ }^{1}$ In both of our patients an anomalous origin of the RCA was suspected at CCA, but no further information about the vessels was given.

CTCA allowed a detailed evaluation of the course and the caliber of anomalous vessels, proving their "malignant nature" due to the inter-arterial course between the aorta and the pulmonary trunk, not seen at CCA (Fig. 2a). Two other details associated with the "malignant nature" of the coronary anomaly were easily detectable in our patients by means of CTCA maximum intensity projection image (MIP) and 3D intraluminal view: an acute take-off angle at the juncture to the left coronary sinus (Fig. 2c) and a slit-like coronary orifice caused by the compression of the proximal portion of the RCA (Figs. 1d and 2d). In particular, the 3D intraluminal view reconstruction can reveal the relationship between the leaflets of the aortic valve and the ostium of the RCA, emphasizing its oval shape, which is a crucial detail for pre-operative evaluation. ${ }^{4}$

Management of malignant coronary anomalies is controversial. In the last 40 years the management strategy has shifted from predominantly medical, with long-term $\beta$-blocking therapy, to almost exclusively surgical. ${ }^{5,6}$ Surgical treatment, nowadays, is generally recommended, but some authors do not suggest this approach for all patients. ${ }^{6}$ Surgery is indicated in young patients, patients with very active lifestyle, athletes, or 
patients with proven ischemia during stress tests. Multiple surgical options, mainly based on the different morphology of the coronary anomaly (ostial status and intramural course), are available. They include coronary artery bypass graft, coronary reimplantation, unroofing of the intramural segment (also named ostioplasty), and pulmonary artery translocation. ${ }^{7,8}$ Coronary artery bypass grafting without ligating the anomalous RCA has been advocated as the surgical strategy of choice for over a decade but, in absence of occlusive disease, there are concerns about competitive flow. ${ }^{8}$ Percutaneous treatment with stent implantation has been proposed, but it is not generally accepted. ${ }^{9}$

\section{REFERENCES}

1. Kim SY, Seo JB, Do KH, et al: Coronary artery anomalies: Classification and ECG-gated multi-detector row CT findings with angiographic correlation. Radiographics 2006;26: 317-334.

2. Chang HY, Yin WH: Anomalous right coronary artery arising from left coronary sinus in two brothers. Acta Cardiol Sin 2011;27:124-127.
3. Montaudon $\mathrm{M}$, Latrabe $\mathrm{V}$, Iriart $\mathrm{X}$, et al: Congenital coronary artery anomalies: Review of the literature and multi detector computed tomography (MDCT)-appearance. Surg Radiol Anat 2007;29:343-355.

4. Baird CW: Intraluminal 3-D CT identifies coronary osteal and commisural relationships in anomalous aortic origin of coronary arteries. Ann Thorac Surg 2010;89:1339-1345.

5. Krasuski RA, Magyar D, Hart S, et al: Long-term outcome and impact of surgery on adults with coronary arteries originating from the opposite coronary cusp. Circulation 2011;123:154-162.

6. Zemanek D, Vaselka J: Coronary artery anomalies-A short review. CEJ Med 2007;2(2):140-153.

7. Davies JE, Burkhart HM, Dearani JA, et al: Surgical management of anomalous aortic origin of a coronary artery. Ann Thorac Surg 2009;88(3):844-848.

8. Gulati R, Reddy VM, Culbertson C, et al: Surgical management of coronary artery arising from the wrong coronary sinus using standard and novel approaches. J Thorac Cardiovasc Surg 2007;134:1171-1178.

9. Hariharan R, Kacere RD, Angelini P: Can stent-angioplasty be a valid alternative to surgery for anomalous origination of a coronary artery. Tex Heart Inst J 2002;29(4):308313. 